This item was submitted to Loughborough's Research Repository by the author.

Items in Figshare are protected by copyright, with all rights reserved, unless otherwise indicated.

\title{
A new adaptive blind equaliser structure with robustness to loss of channel disparity
}

PLEASE CITE THE PUBLISHED VERSION

PUBLISHER

(c) IEEE

VERSION

VoR (Version of Record)

LICENCE

CC BY-NC-ND 4.0

\section{REPOSITORY RECORD}

Skowratananont, K., Sangarapillai Lambotharan, and Jonathon Chambers. 2019. "A New Adaptive Blind Equaliser Structure with Robustness to Loss of Channel Disparity". figshare. https://hdl.handle.net/2134/5815. 
This item was submitted to Loughborough's Institutional Repository (https://dspace.lboro.ac.uk/) by the author and is made available under the following Creative Commons Licence conditions.

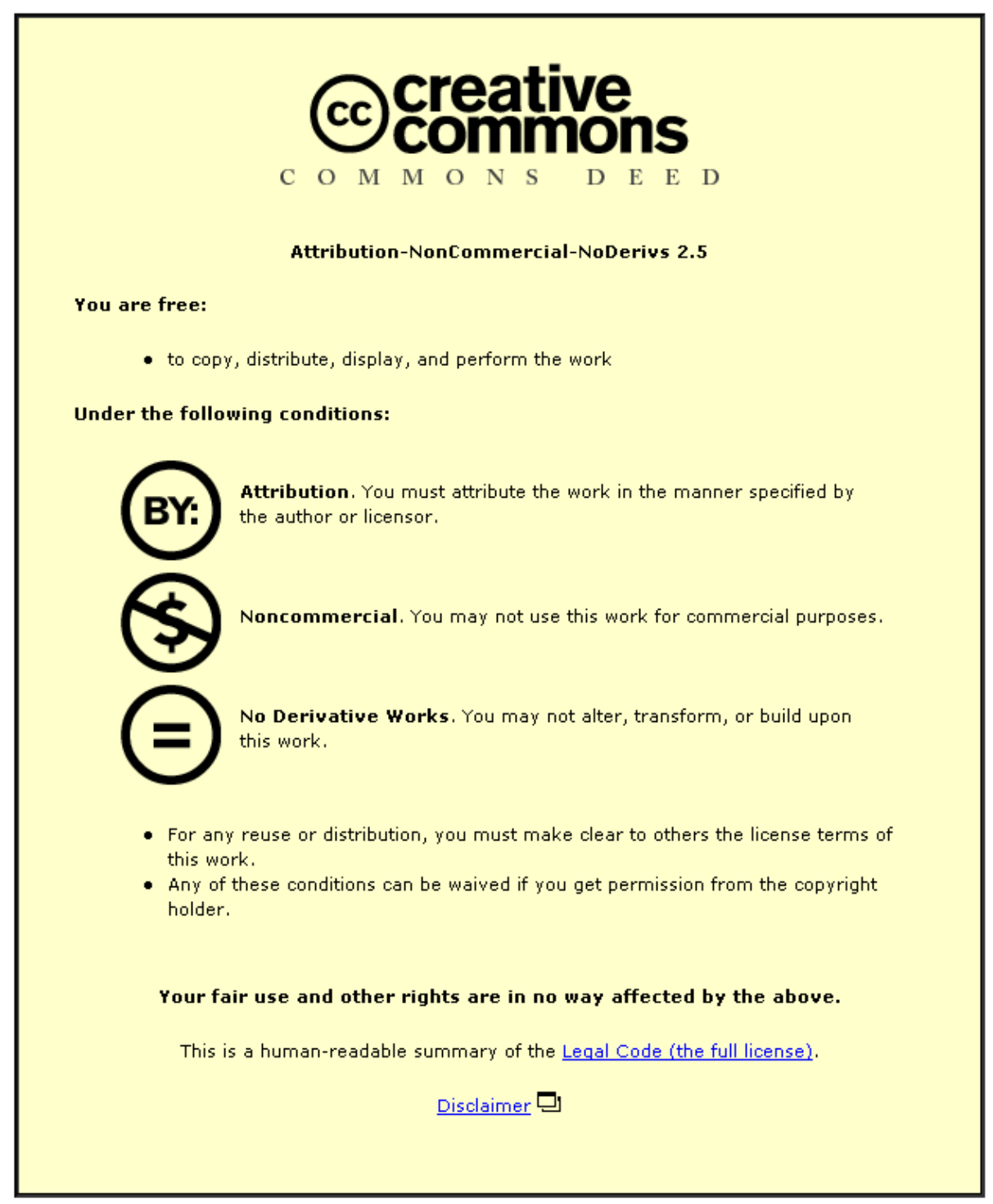

For the full text of this licence, please go to: http://creativecommons.org/licenses/by-nc-nd/2.5/ 


\title{
A NEW ADAPTIVE BLIND EQUALISER STRUCTURE WITH ROBUSTNESS TO LOSS OF CHANNEL DISPARITY
}

\author{
K. Skowratananont, S. Lambotharan, and J.A. Chambers \\ Signal Processing and Digital Systems Section \\ Department of Electrical and Electronic Engineering \\ Imperial College, London, SW7 2B'T \\ E-mail: k.skowratananont@ic.ac.uk
}

\begin{abstract}
We address the problem of blind Fractionally Spaced Channel (FSC) identification/equalisation when channel disparity is lost. With an extension of the EigenVector based AlgorithM (EVAM), we show that it is possible to identify the uncommon part of an FSC. The new blind Decision Feedback Equaliser (DFE) structure therefore first equalises the uncommon part of the channels with a fractionally spaced feedforward equaliser, and then uses a baud spaced DFE to equalise the common part of the channels. We also demonstrate that this new equaliser structure is appropriate for difficult channels, with non minimum phase zeros close to the unit circle. Simulation studies are included to support the work.
\end{abstract}

\section{INTRODUCTION}

Second order statistics (SOS) based blind fractionally spaced channel (FSC) identification algorithms have received, by far, the most attention in the literature. Techniques such as the subspace method proposed in [2] and the time domain method proposed in [1] perform well, even under low SNR and time varying channel conditions [5]. Unfortunately, when the FSC contains common zeros in the subchannels and when the order of the channel is unknown, the performance of these techniques degrades drastically. It has been shown in [4] that the subspace method is capable of identifying only the uncommon part of the FSC (up to a scalar factor). Another technique, which performs similarly to the subspace method, is the EVAM method in [3]. Extension to these techniques in order to obtain the true channel coefficients will be introduced.

In certain circumstances, the parameters of interest are not the channel coefficients, but only the retrieval of the transmitted signal. Several methods based on SOS then need to be further linked with a fractionally spaced equaliser (FSE) to obtain the estimate of the input sequence. In the absence of channel noise, the FSC without common zeros can be completely equalised with the zero forcing FSE. However, with the presence of channel noise, as the estimated uncommon zeros of the FSC possibly approach one another, the noise amplification factor of the minimum mean squared error (MMSE) equaliser, as well as the mean squared error (MSE) at the equaliser output, increase significantly.

In this paper, we propose a new decision feedback equaliser (DFE) which is robust to both the lack of channel disparity and the over estimated channel order.

\section{EVAM FOR THE IMPERFECT CHANNEL}

In this section, we investigate the robustness with respect to the lack of the channel disparity of the EVAM method. For simplicity, the investigation will be for the two subchannels case with the subchannels of order $M$. However, this can be extended readily for any numbers of subchannels. The EVAM framework for multichannel identification is depicted in Figure 1. $W_{j}\left(z^{-1}\right)=$ $\sum_{i=0}^{N-1} w_{j}(i) z^{-i} j=0,1$ denotes the FIR filter of length $N$ for the output of subchannel $H_{j}\left(z^{-1}\right)$. The polynomials $W_{0}\left(z^{-1}\right)$ and $W_{1}\left(z^{-1}\right)$ can be represented as a vector $\mathbf{W}=\left[w_{0}(1), w_{1}(1), \ldots, w_{0}(N), w_{1}(N)\right]^{T}$, and then, $e(k)=\mathbf{W}^{T} Y(k)$ where $Y(k)$ is the channel output vector. By minimising $E\left\{e^{2}(k)\right\}$, the polynomials $W_{0}\left(z^{-1}\right)$ and $W_{1}\left(z^{-1}\right)$ are given as

$$
\begin{aligned}
& W_{0}\left(z^{-1}\right)=\alpha H_{1}\left(z^{-1}\right) \\
& W_{1}\left(z^{-1}\right)=-\alpha H_{0}\left(z^{-1}\right)
\end{aligned}
$$

where $\alpha$ is an arbitrary constant if $N$ is equal to $M+1$. However, in practice, the channel order is difficult to estimate. This results in an over estimate of the channel order $(N>M+1)$. Due to the over-estimation, the 


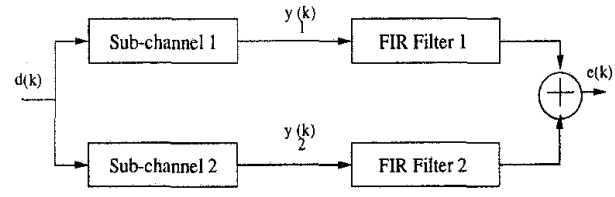

Figure 1: EVAM blind channel identification framework.

$\alpha$ in (1) becomes an arbitrary transfer function with order of $N-M-1$, and the roots of the FSC will be defined from the roots of $W_{0}\left(z^{-1}\right)$ and $W_{1}\left(z^{-1}\right)$ which are at different locations.

Next we consider the case when the FSC lacks disparity. The subchannels, $H_{j}\left(z^{-1}\right)$, are given by,

$$
H_{j}\left(z^{-1}\right)=k_{j} U_{j}\left(z^{-1}\right) C\left(z^{-1}\right), j=0,1
$$

where $U_{j}\left(z^{-1}\right)=1+\sum_{k=1}^{M_{u}} u_{j}(k) z^{-k}$ and $C\left(z^{-1}\right)=$ $1+\sum_{k=1}^{M_{c}} c(k) z^{-k}$ correspond respectively to the uncommon part of the FSC and the common part of the FSC. The parameter $k_{j}$ is the first coefficient of $H_{j}\left(z^{-1}\right)$, (ie. $\left.h_{j}(0)\right)$. Let us assume that $M_{c}$ is small and the order of the subchannels, $M=M_{u}+M_{c}$. When the common zeros exist in FSC, the polynomials, $W_{0}\left(z^{-1}\right)$ and $W_{1}\left(z^{-1}\right)$, become

$$
\begin{aligned}
& W_{0}\left(z^{-1}\right)=\beta k_{1} A\left(z^{-1}\right) U_{1}\left(z^{-1}\right) \\
& W_{1}\left(z^{-1}\right)=-\beta k_{0} A\left(z^{-1}\right) U_{0}\left(z^{-1}\right)
\end{aligned}
$$

where the $\beta$ is an arbitrary constant and $A\left(z^{-1}\right)=$ $1+\sum_{k=1}^{N-M_{u}-1} a_{j}(k) z^{-k}$ is an arbitrary transfer funetion. This implies that the roots of uncommon part of FSC remain identified from the different root locations between $W_{0}\left(z^{-1}\right)$ and $W_{1}\left(z^{-1}\right)$. Notice that to design the proposed DFE, it is very important to estimate the ratios $\frac{k_{0}}{k_{1}}, \ldots, \frac{k_{0}}{k_{(L-1)}}$ where $L$ is the number of subchannels. We consider that the first coefficients of polynomials $W_{0}\left(z^{-1}\right)$ and $W_{1}\left(z^{-1}\right)$ in $(3)$ are $\beta k_{1}$ and $-\beta k_{0}$, respectively. We therefore have the ratio as

$$
\frac{k_{1}}{k_{0}}=-\frac{W_{0}(1)}{W_{1}(2)}
$$

Moreover, what we need to know is $k_{0}$ and $k_{1}$ which enable us to find the true channel coefficients of $H_{0}\left(z^{-1}\right)$ and $H_{1}\left(z^{-1}\right)$. This problem will be explained in more detail in section 4 .

\section{A NEW DFE FOR THE LACK OF CHANNEL DISPARITY AND THE UNKNOWN ORDER PROBLEMS}

Exploiting the knowledge of the uncommon part, a feed forward FSE is designed so that the combination of the uncommon part of the FSC and FSE is a pure delay. Indeed, the combination of the FSC and FSE is the common part of the FSC up to a scaling factor, see Figure 2 for $L=2$.

Now consider the common part of the FSC whose magnitude response is equalised with an IIR filter. Therefore, the IIR filter is adapted to minimise $J(r)=E\left\{p^{2}(k)\right\}$ where $p(k)$ is the output of the IIR filter. The IIR filter is adapted according to $\mathbf{r}_{k+1}=\mathbf{r}_{k}+2 \mu p(k) \mathbf{p}_{k}$ where $\mathbf{p}_{k}=[p(k-1), p(k-2), \cdots, p(k-N r)][6]$. The cost function, $J(r)=E\left\{p^{2}(k)\right\}$, is unimodal, if the order of the IIR filter, $N r$, is equal to or greater than that of the common part of the FSC, $M_{\mathcal{c}}$, [7]. At the minimum the denominator polynomial of the IIR filter, $S\left(z^{-1}\right)$, will be the Spectrally Equivalent Minimum Phase (SEMP) of the common part of the FSC.

$$
I\left(z^{-1}\right)=\frac{1}{\prod_{i=1}^{N_{r}}\left(1-\xi_{i} z^{-1}\right)}=\frac{1}{S\left(z^{-1}\right)}
$$

From the poles of the IIR filter, $2^{M_{c}}$ polynomials, all of which have SEMP, $S\left(z^{-1}\right)$, can be constructed. From these polynomials, $2^{M_{c}}$ DFEs are designed. For each DFE, the squared error is calculated from the error between the input and output of the decision device (see Figure 2 for $M_{c}=1$ ). The output of the decision device corresponding to the minimum squared error will be the estimate of the transmitted signal. The coefficients of this DFE also provide the impulse response of the common part of the FSC.

\section{SCALING FACTOR}

From the previous section, the channel can be equalised completely, only if the first coefficient of the channel, $h_{0}=1$. This is because the first coefficient of the DFE is always assumed to be unity. Unfortunately, in practice, the coefficients of the channel are arbitrary and unknown. Therefore, we need to find some constant, $\mathrm{G}$, to balance the weight between the channel side and the equalisation side. As shown in Figure 2, $G_{i}$ multiplies the input of each DFE. Without prior knowledge of the channel, $\mathrm{G}$ is given by,

$$
G=\sqrt{\frac{\sum(D F E \operatorname{coe} f)^{2}}{\sum_{i=0}^{M_{c}} c_{i}^{2}}}=\sqrt{\frac{\sum(D F E \text { coef })^{2}}{E\left\{x^{2}(k)\right\}}}
$$




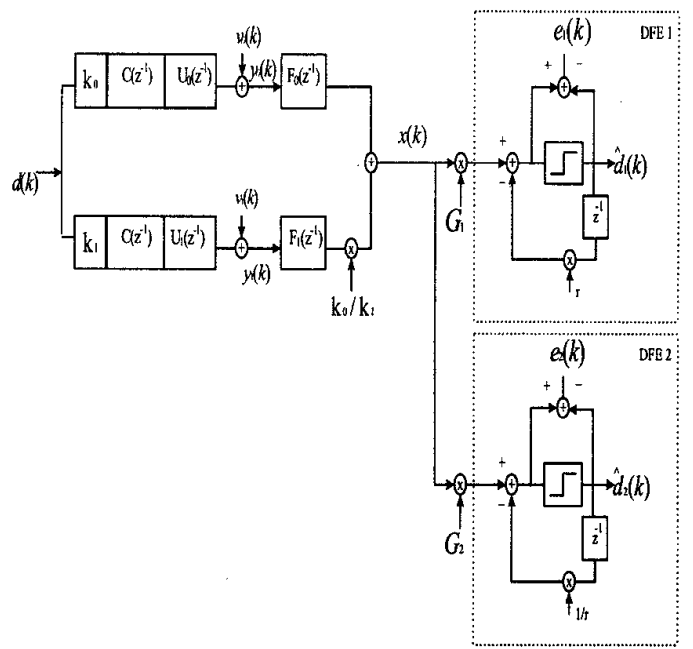

Figure 2: The FSC with a common zero, a FSE, the multiple DFE and the scaling factor, $\mathrm{G}$

where $D F E$ coef and $x(k)$ denote respectively the coefficients of each possible feedback polynomial and the DFE input. (2) can be rewritten as,

$$
\begin{array}{r}
H_{0}\left(z^{-1}\right)=\left[U_{0}\left(z^{-1}\right)\right]\left[k_{0} C\left(z^{-1}\right)\right] \\
H_{1}\left(z^{-1}\right)=\left[\left(k_{1} / k_{0}\right) U_{0}\left(z^{-1}\right)\right]\left[k_{0} C\left(z^{-1}\right)\right]
\end{array}
$$

Within the second bracket in (7), the coefficients of $C\left(z^{-1}\right)$ will correspond to the feedback coefficients of the minimum squared error DFE. Then, $k_{0}$ can be defined as $k_{0}=1 / G$ where $\mathrm{G}$ corresponds to the minimum squared error DFE.

\section{SIMULATION RESULTS}

The fractionally spaced channel is set to be $H_{0}\left(z^{-1}\right)=$ $2+4.1333 z^{-1}+1.3333 z^{-2}$ and $H_{1}\left(z^{-1}\right)=3+5.6 z^{-1}+$ $z^{-2}$ with the corresponding zeros at $(-0.4,-1.6667)$ and $(-0.2,-1.6667)$, respectively. Assume that there is no noise. By using the EVAM algorithm, the uncommon part of the FSC, $U_{0}\left(z^{-1}\right)=1+0.4 z^{-1}$ and $U_{1}\left(z^{-1}\right)=$ $1+0.2 z^{-1}$, are identified. The ratio is also estimated as $k_{0} / k_{1}=2 / 3$. The zero forcing fractionally spaced equaliser parameters, $F_{0}=-1$ and $F_{1}=2$, are then designed. Due to the difference of the first coefficients in both subchannels, the equaliser output of subchannel 2 is multiplied by the ratio, $k_{0} / k_{1}$ as shown in Figure 2 .
Then, the baud rate output of the fractionally spaced equaliser is passed to the IIR filter. The coefficient of the IIR filter, averaged over 2500 samples, converges to 0.6006. The squared errors of DFE1 and DFE2 are shown in Figure 3 where the minimum squared error corresponds to DFE2. Consequently, from (6), $k_{0}$ is equal to 1.9874. Exploiting (7), the estimated multichannels are,

$$
\begin{aligned}
H_{0}\left(z^{-1}\right) & =\left[\left(1+0.4 z^{-1}\right)\right]\left[(1.9874)\left(1+1.6649 z^{-1}\right)\right] \\
& =\left[1.9874+4.1038 z^{-1}+1.3235 z^{-2}\right] \\
H_{1}\left(z^{-1}\right) & =\left[(1.5)\left(1+0.2 z^{-1}\right)\right]\left[(1.9874)\left(1+1.6649 z^{-1}\right)\right] \\
& =\left[2.9811+5.5595 z^{-1}+0.9926 z^{-2}\right]
\end{aligned}
$$

which correspond very closely to the desired multichannels with the normalised channel coefficient error of 0.0070 .
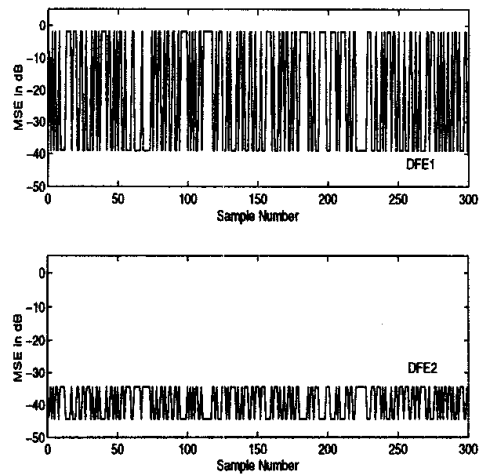

Figure 3: The MSE for DFE1 and DFE2

Next we compare the performance of the proposed algorithm with the fractionally spaced constant modulus algorithm (FS-CMA). This example considers the FSC with the non minimum phase common zero close to the unit circle. The FSC of $H_{0}\left(z^{-1}\right)=1+1.3 z^{-1}+$ $0.22 z^{-2}$ and $H_{1}\left(z^{-1}\right)=1+1.5 z^{-1}+0.44 z^{-2}$ is simulated at $S N R=30 d B$. The FS-CMA equaliser length of 2 is initialised at $F_{0}=[0,1]^{T}$ and $F_{1}=[0,1]^{T}$ which is close to the MMSE equaliser parameter that corresponds to the optimum delay. The squared errors at the equaliser outputs of 1000 samples averaged over 10 independent trails are depicted in Figure 4. Due to the common zero in FSC, we can see that the FS-CMA equaliser provides a relatively high MSE $\approx 0.2453$. With our new technique, the uncommon part of FSC, $1+0.2192 z^{-1}$ and $1+0.4208 z^{-1}$, is estimated by the EVAM algorithm. With the MMSE equaliser, the corresponding FSE is found as [1.9544, -0.9241]. The baud rate output is passed to the IIR filter, and 
the squared errors of the DFE2 (in Figure 2) over 10 independent trails are presented in Figure 4 . The comparative results clearly show the superior mean square error of the new algorithm over the conventional CMA when the FSC lacks of disparity.

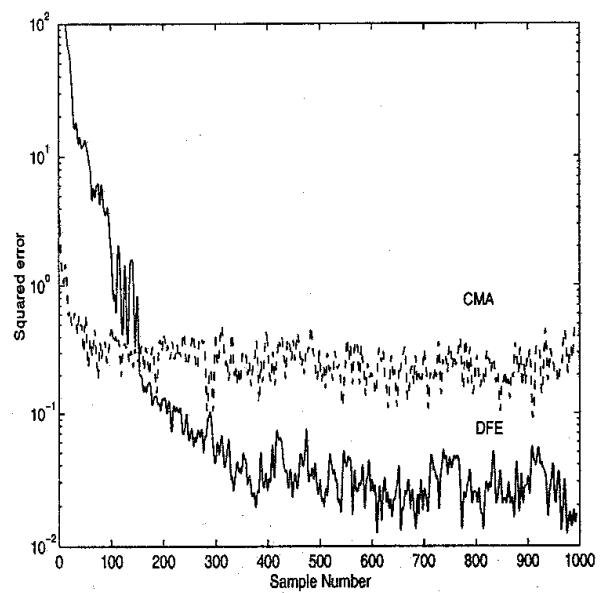

Figure 4: Performance of the CMA and the new DFE algorithms

\section{CONCLUSION}

A new blind decision feedback equaliser for a FSC with common zeros has been proposed. The feedforward section of the proposed equaliser is a FSE which equalises the uncommon part of the FSC, while the feedback section is a DFE which equalises the distortion caused by the common part of the FSC. This technique has been shown to overcome the common zero problem, and to perform well, even with the non minimum phase zero close to the unit circle.

\section{REFERENCES}

[1] Tong L., "Blind identification and equalisation based on second order statistics: a time domain approach," IEEE Trans. Inform. Theory, Vol.40, pp.340-349, 1994.

[2] Moulines E., and Duhamel P., "Subspace methods for the blind identification of multichannel FIR filters," IEEE Trans. Signal Processing, Vol.43, pp.516-525, 1995.

[3] Gurelli M., and Nikias C., "EVAM: an eigenvector based algorithm for multichannel blind deconvolution of input coloured signal," IEEE Trans. Signal Processing, Vol.43, pp.134-149, 1995.
[4] Touzni A. and Fijalkow I., "Robustness of blind fractionally spaced identification/equalisation to loss of channel disparity," ICASSP 97, Munich, pp.3937-3940, 1997.

[5] Skowratananont K, and Chambers J., "A comparison of blind channel estimation and equalisation techniques for a fading environment," Sixth International Conference on Telecommunications, Edinburgh, UK, 1998

[6] Shynk J., "Adaptive IIR filtering," IEEE Acoustic, Speech and Signal Processing Magazine, Vol.6, pp.4-21, 1989.

[7] Astrom K., and Soderstrom T., "Uniqueness of the maximum likelihood estimates of the parameters of an ARMA model," IEEE Trans. on Automatic Control, Vol.AC-19, pp.769-773, 1974.

[8] Da Rocha C.A.F., Macchi O., and Romano J.M.T., "An adaptive nonlinear IIR filter for selflearning equalisation," ITC, Rio-de-Janerio, pp.6$10,1994$.

[9] Lambotharan S., and Chambers J., "A new blind equalisation structure for a deep-null communication channels," IEEE Trans, on Circuits and Systems, Vol.45, pp., 1998. 